$\mathrm{DE}$

M E D I C I N A

T R O P I C A L

$\mathrm{DE}$

S ÃO PAULO

JOURNAL OF THE SÃO PAULO INSTITUTE OF TROPICAL MEDICINE
1 Universidade Estadual de Campinas, Faculdade de Ciências Médicas, Campinas, São Paulo, Brazil

Correspondence to: Marina Rovani Drummond

Universidade Estadual de Campinas, Faculdade de Ciências Médicas, Rua Tessália Vieira Camargo, 126, Barão Geraldo, CEP 13083-887, Campinas, SP, Brazil

Tel: +55 $193521-9134$

E-mail: marina.rovani@gmail.com

Received: 27 February 2019

Accepted: 2 July 2019

\section{Bartonella henselae bacteremia diagnosed post-mortem in a myelodysplastic syndrome patient}

\author{
Marina Rovani Drummond ${ }^{(1)}$, Lorena Visentainer ${ }^{1}$, Amanda Roberta de \\ Almeida ${ }^{1}$, Rodrigo Nogueira Angerami ${ }^{1}$, Francisco Hideo Aoki ${ }^{1}$, Paulo \\ Eduardo Neves Ferreira Velho 1
}

\section{ABSTRACT}

This study involves a 49-year-old male, who for three years suffered with a myelodysplastic syndrome and who needed frequent blood transfusions. One day following a transfusion, he presented fever and abdominal pain. The fever became persistent and only improved temporarily with two cycles of intravenous ciprofloxacin. Nearly 120 days after beginning the second cycle of treatment, he had experienced a weight loss of $16 \mathrm{~kg}$ and recurring fever. Screening for fever of unknown origin was conducted, including Bartonella infection. No etiology could be found. The patient improved with an antimicrobial regimen composed of oral doxycycline and intravenous ciprofloxacin. After 15 days afebrile, the patient was discharged with a four-month oral prescription of doxycycline and ciprofloxacin. Eight months following the antibiotic treatment, the patient received an allogeneic bone marrow transplant. Five days following the transplant, the patient initiated a febrile neutropenia and died. From a blood sample collected and stored at the time of hospitalization, a microbiological and molecular study was performed again. Blood- and liquid culture-PCRs from the same blood sample were all negative, but an isolate from solid subculture was found. The molecular reactions from this isolate were all positive and the sequence was $100 \%$ homologous to Bartonella henselae. The present report points to the limitations of laboratory techniques currently available for investigation of possible cases of bartonellosis in clinical practice, and the potential risk of Bartonella spp. transmission through blood transfusions.

KEYWORDS: Bartonella. Myelodysplastic syndromes. Differential diagnosis. Blood transfusion.

Bartonella spp. are Gram-negative, facultative intracellular bacteria. They are known to infect erythrocytes and endothelial cells, causing chronic and cyclic bacteremia in their hosts. The clinical spectrum of bartonellosis has increased rapidly and infection by these bacteria appears to be much more prevalent than diagnosed ${ }^{1}$.

Although there is no laboratory diagnosis with $100 \%$ of sensitivity and specificity, the greatest limitation to bartonellosis diagnosis is that most physicians do not consider this hypothesis. Any patient with a history of unknown etiology of prolonged fever, recurrent or severe anemia, febrile maculopapular rash, hepatitis or chronic lymph node disease should include the differential diagnosis of bartonellosis ${ }^{2,3}$.

This report describes a man with myelodysplastic syndrome who experienced fever and abdominal pain following a blood transfusion. Bartonellosis was confirmed after isolation of bacteria in solid microbiological culture. 


\section{CASE REPORT}

A 49-year-old male electrical engineering technician

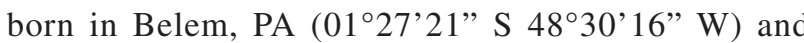
living in Porto Velho, RO ( $8^{\circ} 45^{\prime} 42^{\prime \prime} \mathrm{S} 63^{\circ} 54^{\prime} 14^{\prime}$ 'W), Brazil, was referred to the Hematology Division of the University of Campinas (UNICAMP) Hospital, Campinas, SP (2254'25.5744" S 47³'47.6640” W) due to pancytopenia. He reported having undergone aortic valve replacement, pulmonary valve graft, and definitive pacemaker implantation by total atrioventricular block seven years earlier. He did not provide details of his medical conditions but denied having had fever at that time. Three years prior to this exam, thrombocytopenia had been detected in a routine blood count $\left(81,000 \mathrm{cells} / \mathrm{mm}^{3}\right.$ of blood), with no hemorrhagic manifestations. His condition was accompanied regularly in Porto Velho. During this period he developed pancytopenia, and underwent transfusions of red blood cells every 15 to 30 days. He denied smoking, alcoholism and the use of illicit drugs. He reported contact with dogs and denied contact with cats. After evaluation at the Hematology Division, the initial hypothesis of myelodysplastic syndrome was confirmed by a bone marrow biopsy.

Eight months after the diagnosis, the patient needed another transfusion of red blood cell concentrate, which was performed in another hospital. The day after the transfusion procedure, the patient had an episode of fever, abdominal pain and vomiting. The febrile condition persisted for approximately ten days and, after a transient improvement, he again presented persistent fever associated with hypogastric colic, but without changes in bowel and urinary habits or respiratory complaints. The patient was then admitted to the same hospital in which he had undergone the last transfusion procedure to investigate the febrile condition. Blood cultures collected during hospitalization were negative. However, abdominal computed tomography revealed a high level of renal discharge, suggesting a renal infection, in spite of a negative urine culture. Nevertheless, a pyelonephritis diagnostic hypothesis was considered without microbiological confirmation, and an antimicrobial fourteen-day treatment with parenteral ciprofloxacin was started.

Soon after discharge, the patient was hospitalized again due to fever of unknown origin (FUO). Examinations included transesophageal echocardiography (which did not show signs of valvular vegetation), a gastrointestinal endoscopy and a colonoscopy (which also showed no alterations). He was treated with a new 14-day course of parenteral ciprofloxacin. He evolved afebrile and remained so for 35 days.
Four months after the onset of fever, the patient returned to the Hematology Division at UNICAMP Hospital seeking medical attention, for a five-day fever. The fever was persistent, with temperatures reaching $40{ }^{\circ} \mathrm{C}$ at night, accompanied by a drop in his general condition. He reported chills, sweating and low back pain. He had lost $16 \mathrm{~kg}$ since the initial febrile condition five months earlier. He did not present respiratory or urinary complaints. Upon physical examination, with the exception of anemia and a panfocal cardiac murmur related to the valve replacement, there were no other changes or clinical signs. Laboratory results presented hematuria with erythrocytic dismorphysm, in addition to pancytopenia. The patient was hospitalized at the UNICAMP Hospital for a new FUO investigation with hypotheses of bacterial endocarditis, renal tuberculosis, malaria, salmonellosis, visceral leishmaniasis, brucellosis and bartonellosis.

In the laboratory investigation, serological exams for hepatitis B and C, HIV, syphilis, toxoplasmosis, Epstein-Barr virus, cytomegalovirus, Chagas' disease, schistosomiasis, brucellosis, coxiellosis and bartonellosis showed no evidence of acute or recent infections. There were no parasites in peripheral blood smears. Ten blood cultures and three urine cultures, conventional and specific for mycobacteria and for fungi were performed. All results were negative. Direct searches and cultures of the bone marrow aspirates were negative for leishmania, fungi and mycobacteria. In order to detect a possible Bartonella sp. infection, conventional PCR was performed for the ITS region $^{4}$ and nested PCR was performed for the ftsZ gene ${ }^{5}$ from a blood sample whose DNA was extracted using a QIAamp DNA minikit (Qiagen Inc., USA), and the results were also negative. Full-body scintigraphy using gallium showed hypo captive lesions in both kidneys which, in association with glomerular hematuria and a febrile consumption syndrome, reinforced the hypothesis of renal tuberculosis. However, a renal biopsy showed negative culture for mycobacteria and a non-specific histology.

After fourteen days of hospitalization, without diagnosis and considering the previous contact with dogs, an antimicrobial regimen was initiated with oral doxycycline and intravenous ciprofloxacin. The patient remained febrile for four days, after which the antimicrobial regimen was changed to piperacillin/sulbactam and vancomycin. No fever appeared the following day, but after five days he had higher daily fever spikes again. One week later, doxycycline and ciprofloxacin were reintroduced, given that the patient became afebrile less than $24 \mathrm{~h}$ after the change of the antimicrobial regimen. After being afebrile for fifteen days, the patient was discharged with a prescription of doxycycline and ciprofloxacin. He remained asymptomatic 
for two months after which he experienced a five-day fever episode at home, in spite of having no change in the use of antibiotics. There followed a spontaneous improvement of fever and the antibiotics were suspended four months after discharge.

Eight months later, without antibiotics, the patient was submitted to an allogeneic full match bone marrow transplantation (the donor being his sister), due to the underlying disease. Five days after transplantation, the patient initiated a febrile neutropenia, and an empiric treatment with piperacillin/sulbactam, vancomycin and metronidazole was initiated. At the time, blood and urine cultures were collected and they were all negative. However, the patient remained febrile even during antibiotic therapy. Ten days after transplantation, he developed a septic shock, followed by death. Figure 1 synthesizes the patient's medical history.

Thereafter, a liquid culture was performed using a blood sample collected and stored during the hospitalization that preceded the transplantation, and during which the diagnostic hypothesis of bartonellosis was considered. The hypothesis was based on epidemiological contact with dogs and the differential diagnosis of FUO. The liquid medium was based on Maggi et al. ${ }^{6}$ and implemented as follows: 900
$\mathrm{mL}$ of IPL-41 medium (insect medium), $0.1 \mathrm{mg}$ of NAD (nicotinamide adenine dinucleotide), $1.25 \mathrm{mg}$ of NADP (nicotinamide adenine dinucleotide phosphate), $2 \mathrm{mg}$ of ATP (adenosine triphosphate), $2 \mathrm{mg}$ of pyruvate and $2 \mathrm{~g}$ of yeast extract. Two milliliters of whole blood (collected in EDTA tube) were introduced into $8 \mathrm{~mL}$ of liquid medium. Liquid cultures were maintained under constant agitation at $35{ }^{\circ} \mathrm{C}$ with $5 \% \mathrm{CO}_{2}$ for $14 \mathrm{~d}$. Then a subculture was sown in solid medium ${ }^{3}$ and incubated at $35{ }^{\circ} \mathrm{C}$ with $5 \%$ $\mathrm{CO}_{2}$. New molecular reactions (conventional PCRs for the ITS region, $s s r A$ gene and nested PCR for the fts $Z$ gene) were performed using the stored whole blood sample, and DNA from the liquid culture, extracted using a QIAamp DNA minikit (Qiagen Inc., USA). Table 1 shows the primer sequences used. All PCRs were negative, but there was a microorganism isolated in the solid medium. The conventional PCRs for the ITS region, $s s r A^{7}$ and nested PCR for the $f t s Z^{5}$ from the solid culture isolate were positive. The amplicon from the nested PCR was sequenced and was $100 \%$ homologous to Bartonella henselae Houston-I (access number HG965802.1). Table 2 summarizes the results obtained. All negative controls from each step of the PCR process (DNA extraction, culture and master mix) remained negative.

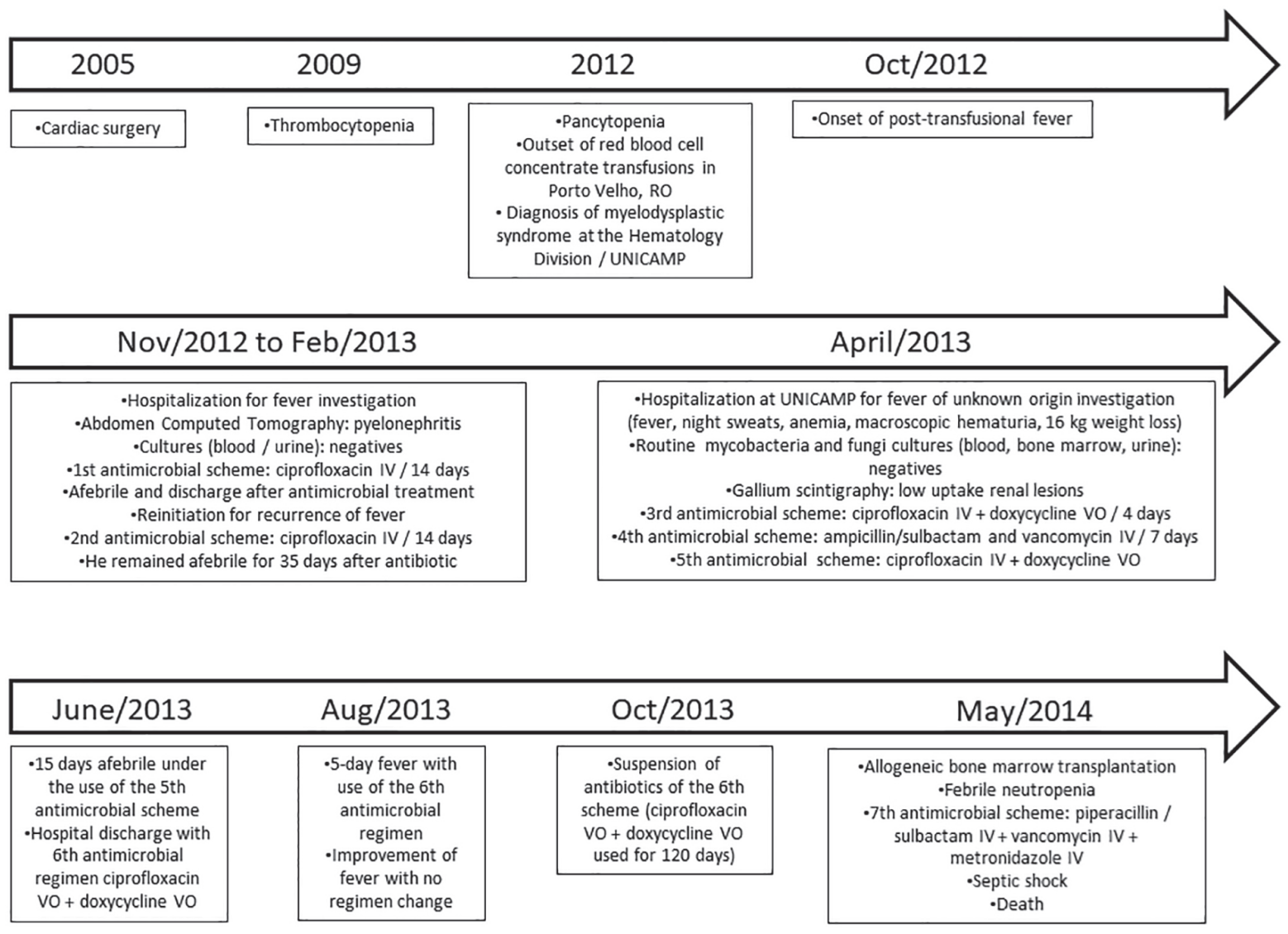

Figure 1 - Timeline illustrating the patient's medical history. 
Table 1 - PCR primers used in the molecular investigation of the patient with a final diagnosis of bartonelosis.

\begin{tabular}{|c|c|c|c|}
\hline Primer & Nucleotide sequence $\left(5^{\prime}-3^{\prime}\right)$ & Molecular target & Reference \\
\hline ITS F & CTTCAGATGATGATCCCAAGCCTTYTGGCG & \multirow{2}{*}{ 16S-23S rRNA spacer } & \multirow{2}{*}{4} \\
\hline ITS R & GAACCGACGACCCCCTGCTTGCAAAGCA & & \\
\hline $\mathrm{BHF}$ & GCCGCAAAGTTCTTTTCATG & \multirow{4}{*}{$f t s Z$} & \multirow{4}{*}{5} \\
\hline $\mathrm{BHR}$ & AGGTGAACGCGCTTGTATTTG & & \\
\hline $\mathrm{BHS}$ & CAAAACGGTTGGAGAGCGT & & \\
\hline $\mathrm{BHA}$ & CGCCTGTCATCTCATCAAGA & & \\
\hline ssrA F & GCTATGGTAATAAATGGACAATGAAATAA & \multirow{2}{*}{ tmRNA } & \multirow{2}{*}{7} \\
\hline ssra R & GCTTCTGTTGCCAGGTG & & \\
\hline
\end{tabular}

Table 2 - Post mortem diagnosis of Bartonella henselae infection through PCR of samples from a 49-year-old male patient with myelodysplastic syndrome.

\begin{tabular}{lccc}
\hline \multirow{2}{*}{ PCR assay } & \multicolumn{3}{c}{ DNA Sample } \\
\cline { 2 - 4 } & Blood & Liquid Culture & Solid Culture \\
\hline Conventional PCR (ITS) & Negative & Negative & Positive \\
Nested-PCR $($ ftsZ) & Negative & Negative & Positive \\
Conventional PCR $($ ssrA $)$ & NP & NP & Positive \\
Final Result & Negative & Negative & Positive \\
\hline
\end{tabular}

NP: Not Performed

\section{DISCUSSION}

Bartonella henselae was the only microorganism identified during the entire investigation process of FUO, suggesting that bartonellosis was probably the cause of the febrile illness and, possibly, of the evolution to sepsis.

It is noteworthy that $B$. henselae was isolated from a blood sample even after two 14-day cycles of parenteral ciprofloxacin. The patient also had a five-day (quintana) fever even after the use of ampicillin / sulbactam and vancomycin for one week and the use of oral doxycycline and ciprofloxacin for two months. Apparently the agent had not been eradicated. In Carrion's disease, $5 \%$ of patients treated for Oroya fever develop verruga peruana (Peruvian wart) and bacterial clumps can be observed in cutaneous lesions, despite adequate treatment and improvement of fever. In this infection, antibiotic treatment can improve the course of the clinical manifestation, but it may not necessarily promote the eradication of Bartonella bacilliformis $^{8,9}$. In addition, there is no consensus about the best treatment for chronic and systemic infection by $B$. henselae. In cases of endocarditis there is indication of the association of intravenous gentamicin (14 days) and oral doxycycline (42 days) $)^{8,10}$.

A possible persistence of infection could not be ruled out, since in none of the antimicrobial regimens, the association of the recommended drug and the minimum duration proposed were followed.

In addition to demonstrating the possible prolonged persistence of $B$. henselae even after the use of successive antimicrobial regimens, we observed the limitation of the sensitivity of several diagnostic methods available, suggesting that non-reactive serological tests and negativecultures or negative-molecular assays do not allow to rule out the Bartonella sp. infection diagnosis.

In a study with blood donors, it was not possible to demonstrate the association between positive serological tests (indirect immunofluorescence) and Bartonella sp. DNA detection of in blood samples ${ }^{11}$. This may explain the patient negative serology and the positive molecular test. In the same study with asymptomatic donors it was possible to isolate colonies of $B$. henselae in six donors. In five cases, the molecular tests using a liquid enrichment culture sample were negative. The serology of just one of the six bacteremic donors tested positive for $B$. henselae and Bartonella quintana antibodies ${ }^{11}$.

Although we were not able to prove a transfusional Bartonella sp. infection, it seems quite plausible that the patient described here was infected during one of the many transfusion procedures, based on the significant temporal relationship between one of the transfusions and the onset of the febrile illness. In addition, $B$. henselae was the only 
microorganism identified after an extensive laboratory investigation. $B$. henselae has already been considered a pathogen with risk of transmission by transfusion ${ }^{12}$ after blood transfusion experimental infections ${ }^{13}$.

In summary, the present case report points to: 1) a possible association of infection by Bartonella spp.; 2) the potential inefficiency of different antimicrobial regimens to eradicate Bartonella spp.; 3) the limitations of laboratory techniques currently available in clinical practice for the investigation of possible cases of bartonellosis; 4) the potential risk of transmission of Bartonella spp. by the transfusional route; 5) the need for greater attention for pathogens such as Bartonella spp. in the investigation of febrile post-transfusional procedures; 6) the need to improve the screening protocols in blood donations to cover pathogens not currently investigated in a usual way.

\section{ACKNOWLEDGMENTS}

PENFV thanks National Council for Scientific and Technological Development (CNPq) for the Research Productivity Scholarship and MRD thanks CNPq for doctoral scholarship.

\section{CONFLICT OF INTERESTS}

No conflicting financial interests exist.

\section{REFERENCES}

1. Pulliainen AT, Dehio C. Persistence of Bartonella spp. stealth pathogens: from subclinical infections to vasoproliferative tumor formation. FEMS Microbiol Rev. 2012;36:563-99.

2. Florin TA, Zaoutis TE, Zaoutis LB. Beyond cat scratch disease: widening spectrum of Bartonella henselae infection. Pediatrics. 2008;121:e1413-25.

3. Drummond MR, Lania BG, Diniz PP, Gilioli R, Demolin DM, Scorpio DG, et al. Improvement of Bartonella henselae DNA detection in cat blood samples by combining molecular and culture methods. J Clin Microbiol. 2018;56:e01732-17.
4. Diniz PP, Maggi RG, Schwartz DS, Cadenas MB, Bradley JM, Hegarty B, et al. Canine bartonellosis: serological and molecular prevalence in Brazil and evidence of co-infection with Bartonella henselae and Bartonella vinsonii subsp. berkhoffii. Vet Res. 2007;38:697-710.

5. Kawasato KH, Oliveira LC, Velho PE, Yamamoto L, Del Negro GM, Okay TS. Detection of Bartonella henselae DNA in clinical samples including peripheral blood of immune competent and immune compromised patients by three nested amplifications. Rev Inst Med Trop Sao Paulo. 2013;55:1-6.

6. Maggi RG, Duncan AW, Breitschwerdt EB. Novel chemically modified liquid medium that will support the growth of seven bartonella species. J Clin Microbiol. 2005;43:2651-5.

7. Diaz MH, Bai Y, Malania L, Winchell JM, Kosoy MY. Development of a novel genus-specific real-time PCR assay for detection and differentiation of Bartonella species and genotypes. J Clin Microbiol. 2012;50:1645-9.

8. Rolain JM, Brouqui P, Koehler JE, Maguina C, Dolan MJ, Raoult D. Recommendations for treatment of human infections caused by Bartonella species. Antimicrob Agents Chemother. 2004;48:1921-33.

9. Maguina C, Garcia PJ, Gotuzzo E, Cordero L, Spach DH. Bartonellosis (Carrion's disease) in the modern era. Clin Infect Dis. 2001;33:772-9.

10. Raoult D, Fournier PE, Vandenesch F, Mainardi JL, Eykyn SJ, Nash J, et al. Outcome and treatment of Bartonella endocarditis. Arch Intern Med. 2003;163:226-30.

11. Pitassi LH, Paiva Diniz PP, Scorpio DG, Drummond MR, Lania BG, Barjas-Castro ML, et al. Bartonella spp. bacteremia in blood donors from Campinas, Brazil. PLoS Negl Trop Dis. 2015;9:e0003467.

12. Stramer SL, Hollinger FB, Katz LM, Kleinman S, Metzel PS, Gregory KR, et al. Emerging infectious disease agents and their potential threat to transfusion safety. Transfusion. 2009;49 Suppl 2:1S-29S.

13. Magalhães RF, Pitassi LH, Salvadego M, Moraes AM, BarjasCastro ML, Velho PE. Bartonella henselae survives after the storage period of red blood cell units: is it transmissible by transfusion? Transfus Med. 2008;18:287-91. 